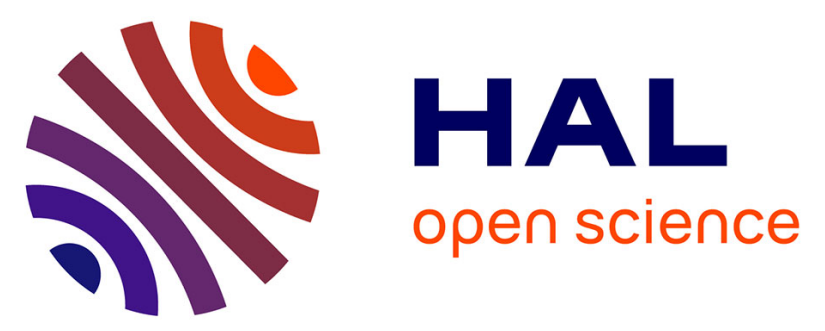

\title{
Thermometry and signatures of strong correlations from Raman spectroscopy of fermionic atoms in optical lattices
}

\author{
Jean-Sebastien Bernier, Tung-Lam Dao, Corinna Kollath, Antoine Georges, \\ Pablo Cornaglia
}

\section{To cite this version:}

Jean-Sebastien Bernier, Tung-Lam Dao, Corinna Kollath, Antoine Georges, Pablo Cornaglia. Thermometry and signatures of strong correlations from Raman spectroscopy of fermionic atoms in optical lattices. Physical Review A : Atomic, molecular, and optical physics [1990-2015], 2010, 81 (6), pp.063618. 10.1103/PhysRevA.81.063618 . hal-00746829

\section{HAL Id: hal-00746829}

https://hal-iogs.archives-ouvertes.fr/hal-00746829

Submitted on 7 Dec 2015

HAL is a multi-disciplinary open access archive for the deposit and dissemination of scientific research documents, whether they are published or not. The documents may come from teaching and research institutions in France or abroad, or from public or private research centers.
L'archive ouverte pluridisciplinaire HAL, est destinée au dépôt et à la diffusion de documents scientifiques de niveau recherche, publiés ou non, émanant des établissements d'enseignement et de recherche français ou étrangers, des laboratoires publics ou privés. 


\title{
Thermometry and signatures of strong correlations from Raman spectroscopy of fermionic atoms in optical lattices
}

\author{
Jean-Sébastien Bernier, ${ }^{1}$ Tung-Lam Dao, ${ }^{2}$ Corinna Kollath, ${ }^{1}$ Antoine Georges, ${ }^{1,3}$ and Pablo S. Cornaglia ${ }^{4}$ \\ ${ }^{1}$ Centre de Physique Théorique, École Polytechnique, CNRS, F-91128 Palaiseau Cedex, France \\ ${ }^{2}$ Laboratoire Charles Fabry de l'Institut d'Optique, CNRS, Université Paris-Sud, Campus de l'École Polytechnique, \\ F-91127 Palaiseau Cedex, France \\ ${ }^{3}$ Collège de France, 11 Place Marcelin Berthelot, F-75005 Paris, France \\ ${ }^{4}$ Centro Atómico Bariloche and Instituto Balseiro, Comisión Nacional de Energia Atómica, Consejo Nacional de Investigaciones \\ Científicas y Técnicas, 8400 Bariloche, Argentina \\ (Received 21 December 2009; published 10 June 2010)
}

\begin{abstract}
A method is proposed to directly measure the temperature of a gas of weakly interacting fermionic atoms loaded into an optical lattice. This technique relies on Raman spectroscopy and is applicable to experimentally relevant temperature regimes. Additionally, a similar spectroscopy scheme can be used to obtain information on the quasiparticle properties and Hubbard bands of the metallic and Mott-insulating states of interacting fermionic spin mixtures. These two methods provide experimentalists with probes to accurately characterize fermionic quantum gases confined to optical lattices.
\end{abstract}

DOI: 10.1103/PhysRevA.81.063618

PACS number(s): 03.75.Ss, 05.30.Fk, 71.10.Li, 71.10.Fd

\section{INTRODUCTION}

Fermionic ultracold-atom physics has witnessed unprecedented experimental progress since a quantum degenerate Fermi gas was first prepared in a three-dimensional optical lattice [1]. The recent evidence for a fermionic Mott-insulating state $[2,3]$ serves as a clear example of these rapid advances. However, despite all these breakthroughs, conducting experiments with fermionic atoms still remains a major challenge as very few probes are available to accurately characterize these systems. Among all the difficulties encountered by experimentalists, the lack of reliable methods to adequately measure the temperature of Fermi gases confined to optical lattices is often cited as one of the main obstacles.

Although this problem is quite persistent when lattice systems are studied, the situation is much better in the continuum, for which several techniques were successfully implemented to estimate the temperature of both fermionic and bosonic quantum gases [4-6]. In the presence of an optical lattice, different schemes to determine the temperature have been devised and experimentally tested for bosonic atoms. One approach relies on the direct comparison of experimental and theoretical time-of-flight images obtained from computationally expensive simulations [7]. In a second method, the temperature is estimated from a measurement of the width of the transition layer between two spin domains created by the application of a magnetic field gradient [8]. Relying on the good local resolution attainable in two-dimensional systems, a third approach extracts temperature from the density [9]. Finally, detecting the temperature using bosonic impurity atoms, which are insensitive to the optical lattice potential, was put forward in [10].

The situation is much more difficult for fermionic atoms loaded into an optical lattice. In current experiments, temperature measurements are usually performed before switching on the lattice potential and after switching it off $[2,3]$. However, since the temperature changes during the loading process, detecting the system temperature with the optical lattice on is of the utmost importance. Experimental attempts [11] at evaluating the temperature of fermions inside a lattice were based on the determination of the number of doubly occupied sites. In this scheme, an accurate evaluation of the temperature requires a full theoretical understanding of the strong dependence of the number of doubly occupied sites with temperature [12-14]. In addition, precise experimental knowledge of the interaction strength, hopping amplitude, trapping configuration, and particle number is needed. The combination of all these requirements renders this approach difficult to use. On the theoretical side, other methods were proposed. For example, one could envisage, as proposed in [15], extracting temperature from a generalized version of the fluctutation-dissipation theorem used in conjunction with the knowledge of both spatially resolved system density and density fluctuations. However, this approach needs sufficiently strong density fluctuations as well as very good local resolution, an experimental requirement that is far from being met for fermionic systems. Finally, in one and two dimensions, measuring the intensity of the light scattered off the atomic lattice array was proposed to detect the system temperature [16].

In this work, we propose a method to measure the temperature of fermions loaded into two- or three-dimensional optical lattices. The approach we put forward relies on transferring a portion of the atoms stored in the optical lattice potential to a third hyperfine state using a stimulated Raman process. Hence, the temperature measurement can be made either locally or globally and can be used in parallel with other probes. Depending on the experimental resolution, this thermometer works for both free and weakly interacting fermions and only requires the knowledge of the hopping amplitude of the system under study. In addition, our approach can be implemented using present fermionic ultracold-atom technology.

Measuring the temperature of fermionic gases loaded into optical lattices is not the only difficulty faced by experimentalists working with cold atoms. Indeed, identifying the different strongly correlated phases that can be realized in these 
systems is also a demanding task. In comparison to condensed matter systems, few probing techniques are available to study cold-atom systems. In the absence of a lattice, spectroscopic methods $[4,5]$, including momentum-resolved radio-frequency spectroscopy [6], were shown to be very efficient in probing the characteristics of fermionic quantum gases. In contrast, measuring the excitation spectrum of gases confined to optical lattices was achieved experimentally only for bosonic systems using Bragg spectroscopy [17-19]. Recently, it was suggested that Raman spectroscopy can also be used to probe the excitation spectrum of strongly correlated phases of Bose gases confined to optical lattices [20,21] and to investigate single-particle excitations in normal and superfluid phases of fermionic gases [22-24]. In this article, we show that Raman spectroscopy can be used to identify various signatures of strongly correlated fermionic phases in optical lattices. Experimentally detectable features include the presence of quasiparticle peaks in weakly and strongly correlated liquids as well as Hubbard bands in strongly correlated liquids and Mott insulators.

The rest of the article is organized as follows: in Sec. II we define the general setup for Raman spectroscopy. In Sec. III we present the temperature detection scheme for noninteracting (Sec. III A) and weakly interacting (Sec. III B) Fermi gases confined to two- and three-dimensional optical lattices. Finally, in Sec. IV we demonstrate that spectroscopy can also be used to identify various signatures of strongly correlated systems.

\section{SETUP AND THEORETICAL DESCRIPTION}

The proposed detection schemes rely on Raman spectroscopy [22,23,25]. This probing technique consists of exciting with a given energy and momentum a many-body state formed of a mixture of two hyperfine states by transferring atoms to a third state. To set the ideas straight, we sketch the Raman process in Fig. 1. There we see that atoms from a hyperfine state $|1\rangle$ are transferred to a different hyperfine state $|3\rangle$ using two Raman laser beams with frequencies $\omega_{12}$ and $\omega_{23}$ and Rabi frequencies $\Omega_{12}$ and $\Omega_{23}$, respectively. The frequencies $\omega_{12}$ and $\omega_{23}$ are both detuned from their corresponding resonances to state $|2\rangle$ to keep this state unoccupied. During the transition, momentum $\mathbf{q}=\mathbf{k}_{1}-\mathbf{k}_{2}$ is transferred to the atoms. This value can be chosen within certain bounds by appropriately adjusting

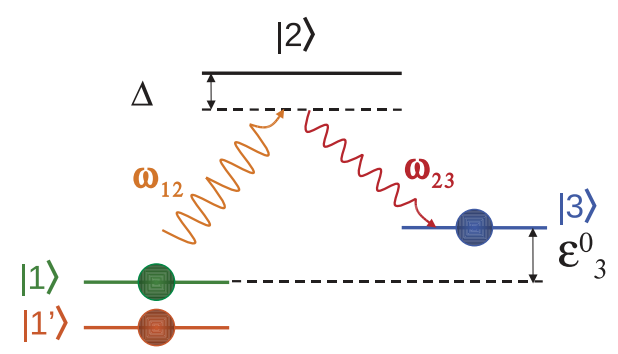

FIG. 1. (Color online) Atomic levels involved in the Raman process. Atoms in hyperfine state $|1\rangle$ are transferred to state $|3\rangle$ using two Raman laser beams with frequencies $\omega_{12}$ and $\omega_{23}$, respectively. These frequencies are both detuned by $\Delta$ from their corresponding resonances to state $|2\rangle$. States $|1\rangle$ and $|3\rangle$ are separated in energy by $\varepsilon_{3}^{o}$.

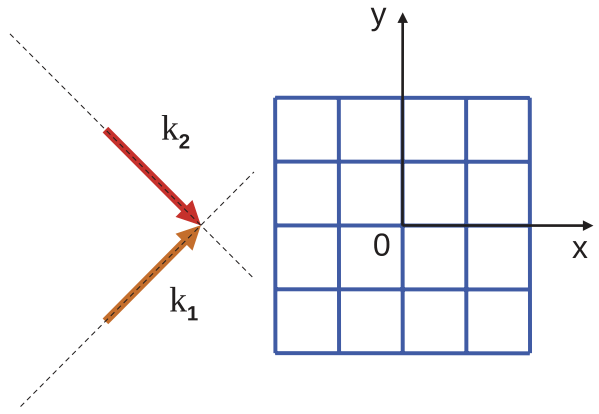

FIG. 2. (Color online) Geometric configuration of the two Raman laser beams carrying momentum $\mathbf{k}_{1}$ and $\mathbf{k}_{2}$, respectively.

the angles between the two Raman beams and the lattice axis (Fig. 2). For example, transferred momentum $\mathbf{q}=0$ could be realized using copropagating Raman laser beams [here, $\mathbf{q}=0$ is shorthand notation for $\mathbf{q}=(0,0)$ or $(0,0,0)]$. However, for many current lattice setups, $\mathbf{q} \approx\left(\frac{\pi}{a}, \frac{\pi}{a}\right)$ or $\left(\frac{\pi}{a}, \frac{\pi}{a}, \frac{\pi}{a}\right)$ could be reached by aligning two counterpropagating Raman lasers along the diagonal of the optical lattice axes.

Experimentally, the Raman signal is measured by counting the number of atoms transferred to state $|3\rangle$. This signal can in principle be resolved both in frequency and momentum. For many applications, such as thermometry, momentum-resolved measurements are not needed. Nevertheless, as we show in Sec. IV, momentum resolution can also provide valuable additional information, but achieving good momentum resolution in optical lattice setups is experimentally demanding.

When only a small fraction of the atoms in hyperfine state $|1\rangle$ are transferred into state $|3\rangle$, the Raman signal can usually be approximated by using a linear response expression [22,23]. Within a local density approximation, the Raman transition rate is given by

$$
\begin{aligned}
R_{\mathbf{q}}(\omega)= & \frac{2 \pi}{\hbar} \sum_{\mathbf{r}} \int d \mathbf{k} W_{\mathbf{k}}^{\mathbf{q}}\left|\Omega_{e}(\mathbf{r})\right|^{2} n_{F}\left(\varepsilon_{3, \mathbf{k}}^{\mathbf{r}}-\hbar \omega-\mu_{o}\right) \\
& \times A\left(\mathbf{k}-\mathbf{q}, \varepsilon_{3, \mathbf{k}}^{\mathbf{r}}-\mu_{o}-\hbar \omega ; \mu_{\mathbf{r}}\right) .
\end{aligned}
$$

In this expression, $\varepsilon_{3, \mathbf{k}}^{\mathbf{r}}=\varepsilon_{3, \mathbf{k}}+V_{3}(\mathbf{r})$ and $\mu_{\mathbf{r}}=\mu_{o}-V_{1}(\mathbf{r})$, where $\varepsilon_{3, \mathbf{k}}$ is the dispersion relation for state $|3\rangle ; V_{1,3}(\mathbf{r})$ are the trapping potentials felt by states $|1\rangle$ and $|3\rangle$, respectively; and $\mu_{o}$ is the chemical potential in the center of the trap. The momentum-dependent coefficient $W_{\mathbf{k}}^{\mathbf{q}}$ is due to the Wannier envelope and is given by

$$
\begin{aligned}
W_{\mathbf{k}}^{\mathbf{q}} & =\left|\int d \mathbf{r} w_{1}^{*}(\mathbf{r}) \psi_{3, \mathbf{k}}(\mathbf{r}) e^{-i \mathbf{q} \cdot \mathbf{r}}\right|^{2} \\
& =\left|\int d \mathbf{r} u_{1, \mathbf{k}}^{*}(\mathbf{r}) u_{3, \mathbf{k}-\mathbf{q}}(\mathbf{r})\right|^{2},
\end{aligned}
$$

where $w_{1}(\mathbf{r})$ is the Wannier function for the atoms in state |1) while $\psi_{3, \mathbf{k}}(\mathbf{r})$ is the Bloch function for the atoms in state $|3\rangle\left(u_{1, \mathbf{k}}, u_{3, \mathbf{k}}\right.$ are the corresponding periodic parts of the Bloch function); $n_{F}(x)=1 /\left\{1+\exp \left[x /\left(k_{B} T\right)\right]\right\}$ is the Fermi function; and $A\left(\mathbf{k}, \hbar v ; \mu_{r}\right)$ is the one-particle spectral function for the $\left(|1\rangle,\left|1^{\prime}\right\rangle\right)$ mixture in a confining potential. The local density approximation has been used, so that $\mu_{\mathbf{r}}$ is the local 
chemical potential at point $\mathbf{r}$. In a homogeneous system, the spectral function is defined as

$$
\begin{aligned}
A(\mathbf{k}, \hbar v)= & \sum_{i, f} \frac{e^{-\bar{E}_{i} / k_{B} T}+e^{-\bar{E}_{f} / k_{B} T}}{\mathcal{Z}} \\
& \times\left|\left\langle\phi_{f}\left|c_{1 \mathbf{k}}\right| \phi_{i}\right\rangle\right|^{2} \delta\left(\hbar v+\bar{E}_{f}-\bar{E}_{i}\right),
\end{aligned}
$$

where $c_{1 \mathbf{k}}$ destroys an atom in state $|1\rangle$ with momentum $\mathbf{k}, \mathcal{Z}=\sum_{i} \exp \left(-\bar{E}_{i} / k_{B} T\right)$ is the grand canonical partition function, the sums over $i$ and $f$ refer to all the many-body states of the system, and the energy $\bar{E}_{i}=E_{i}-\mu N_{i}$ is rescaled by the number of particles. Finally, $\hbar \omega=\hbar\left(\omega_{12}-\omega_{23}\right)$ is the transferred energy, and the Rabi frequency is $\Omega_{e}(\mathbf{r})=$ $\Omega_{12}(\mathbf{r}) \Omega_{23}^{*}(\mathbf{r}) / \Delta$, where $\Delta$ is the detuning. Local resolution of the Raman transfer could be obtained by using a special configuration of laser beams [23].

From Eq. (1), we see that for a given position and momentum the Raman spectrum is obtained from the multiplication of two functions. The first function is the Fermi factor $n_{F}$, which depends strongly on temperature but is independent of other parameters apart from $\mu_{o}$ and $T$ itself. As shown in Sec. III, our temperature detection scheme relies primarily on this observation. The second function entering Eq. (1) is the spectral function, which depends sensitively on the state of the system. We see that this limits somewhat the possibility of a universal temperature determination; but, as explained in Sec. IV, this function provides valuable information on the phase of the system.

Throughout the rest of this article, we describe quantum gases confined to optical lattice potentials using the fermionic Hubbard model [26,27]:

$$
H=-J \sum_{\left\langle r, r^{\prime}\right\rangle \sigma}\left(c_{\sigma r}^{\dagger} c_{\sigma r^{\prime}}+\text { H.c. }\right)+U \sum_{r} \hat{n}_{1 r} \hat{n}_{1^{\prime} r}-\sum_{r \sigma} \mu_{r} \hat{n}_{\sigma r},
$$

where $c_{\sigma r}^{\dagger}$ and $c_{\sigma r}$ are the creation and annihilation operators of the fermions with $\sigma=\left\{|1\rangle,\left|1^{\prime}\right\rangle\right\}, J$ is the hopping matrix element, $U$ is the on-site repulsion, $\mu_{r}$ is the local chemical potential, $\hat{n}_{\sigma r}=c_{\sigma r}^{\dagger} c_{\sigma r}$ is the number operator on site $r$, and $\left\langle r, r^{\prime}\right\rangle$ denotes neighboring lattice sites.

\section{TEMPERATURE DETERMINATION}

In this section, we present a method to evaluate the temperature of weakly interacting fermions confined to optical lattices by measuring their Raman spectrum. We first present this method considering noninteracting fermions in two- and three-dimensional optical lattices. Afterward, we demonstrate how this procedure is also applicable to weakly interacting fermionic gases loaded into optical lattices.

\section{A. Temperature extraction for noninteracting fermions}

For the case of noninteracting fermions (i.e., $U=0$ ), the spectral function for trapped $|1\rangle$ atoms is $A\left(\mathbf{k}, \hbar v ; \mu_{r}\right)=$ $\delta\left(\hbar v+\mu_{r}-\varepsilon_{1, \mathbf{k}}\right)$. In our study, we assume that both $|1\rangle$ and $|3\rangle$ atoms are trapped by the same harmonic potential (i.e., $V_{1}=V_{3}=V_{T}$ ) and are loaded into an optical lattice which is felt equally by both hyperfine states (i.e., induces the same hopping coefficients), such that $\varepsilon_{1, \mathbf{k}}=\varepsilon_{3, \mathbf{k}}-\varepsilon_{3}^{o} \equiv \varepsilon_{\mathbf{k}}$. Here


FIG. 3. (Color online) Left: Raman transfer with $\mathbf{q}=0$; atoms can only be transferred from state $|1\rangle$ to state $|3\rangle$ with frequency $\omega=$ $\varepsilon_{3}^{o} / \hbar$. Right: Raman transfer with $\mathbf{q}=\pi / a$; atoms can in principle be transferred from state $|1\rangle$ to state $|3\rangle$ with frequencies ranging from $\left(\varepsilon_{3}^{o}-2 D\right) / \hbar$ to $\left(\varepsilon_{3}^{o}+2 D\right) / \hbar$, where $D$ is the half-bandwidth; $\varepsilon_{1, \mathbf{k}}$ and $\varepsilon_{3, \mathbf{k}}$ are the dispersions of states $|1\rangle$ and $|3\rangle$, respectively; $\mu$ is the chemical potential for the mixture of states $|1\rangle$ and $\left|1^{\prime}\right\rangle$; and $\varepsilon_{3}^{o}$ is the energy shift of $|3\rangle$ compared to $|1\rangle$.

$\varepsilon_{3}^{o}$ is the energy offset of state $|3\rangle$ with respect to state $|1\rangle$ (cf. Fig. 1). These assumptions are valid as long as we use adequate hyperfine states and confine the atoms into far-detuned optical lattices. Under these conditions, the Raman transition rate is given by

$$
\begin{aligned}
R_{\mathbf{q}}(\omega)= & \frac{2 \pi}{\hbar} \sum_{r} \int d \mathbf{k} W_{\mathbf{k}}^{\mathbf{q}}\left|\Omega_{e}(\mathbf{r})\right|^{2} \\
& \times n_{F}\left(\varepsilon_{3}^{o}-\hbar \omega+\varepsilon_{\mathbf{k}}+V_{T}(\mathbf{r})-\mu_{o}\right) \\
& \times \delta\left(\varepsilon_{3}^{o}-\hbar \omega+\varepsilon_{\mathbf{k}}-\varepsilon_{\mathbf{k}-\mathbf{q}}\right) .
\end{aligned}
$$

This expression depends on temperature only through the Fermi function $n_{F}$. Therefore, detecting the system temperature can be done reliably by fitting $R_{\mathbf{q}}(\omega)$ with a minimum of parameters. From Eq. (5), one also sees that the frequency spread of the Raman signal is strongly dependent on the chosen transferred momentum, q. As shown in Fig. 3, for a homogeneous system, atoms can only be transferred from state $|1\rangle$ to state $|3\rangle$ with energy $\hbar \omega=\varepsilon_{3}^{o}$ if $\mathbf{q}=0$. Hence, $R_{\mathbf{q}=0}(\omega)$ is strongly peaked at $\varepsilon_{3}^{o} / \hbar$ and zero everywhere else [28]. This feature makes it impossible to detect the system temperature using $\mathbf{q}=0$ because the Raman signal is too narrow in $\omega$. What we need is a Raman signal that is nonzero for a wide range of frequencies. This is achieved if $\mathbf{q}=\left(\frac{\pi}{a}, \frac{\pi}{a}\right)$ for square lattices and $\mathbf{q}=\left(\frac{\pi}{a}, \frac{\pi}{a}, \frac{\pi}{a}\right)$ for cubic lattices. As we can see in Fig. 3, for these two configurations, since $\varepsilon_{\mathbf{k}-\mathbf{q}}=-\varepsilon_{\mathbf{k}}$, atoms can in principle be transferred with energies ranging from $\varepsilon_{3}^{o}-2 D$ to $\varepsilon_{3}^{o}+2 D$, where $D$ is the half-bandwidth. Therefore, temperature measurements can be done with this choice of transferred momentum. We use the shorthand notation $\mathbf{q}=\pi / a$ to specify that we use these configurations. We also note that since we are interested in the momentum-integrated Raman rate, $W_{\mathbf{k}}^{\mathbf{q}=\pi / a}$ can be neglected without loss of generality for sufficiently deep lattices as this factor only affects the overall signal amplitude [29].

In the remainder of this section, we demonstrate that Raman spectroscopy can adequately be used as a thermometer. We conduct this demonstration in two steps. First, using Eq. (5), we numerically simulate a Raman experiment in which the probing lasers are shone on the whole system of atoms confined to a lattice and trapped in a harmonic potential. These simulations show that the resulting spectra vary significantly with temperature. Then, as a second step, we fit the obtained 
signal using two different fitting functions. The first is the full continuum-space expression for the Raman rate (valid for large systems):

$$
\frac{R_{\pi / a}(\tilde{\omega})}{N} \propto \frac{C}{\rho} g_{v}\left(\frac{\hbar \tilde{\omega}}{2}\right) \int_{-\infty}^{\mu_{o}} d \mu \frac{\left(\mu_{o}-\mu\right)^{(d-2) / 2}}{1+e^{-(\hbar \tilde{\omega} / 2+\mu) /\left(k_{B} T\right)}} .
$$

In this expression, $\hbar \tilde{\omega}=\hbar \omega-\varepsilon_{3}^{o}, g_{v}(\varepsilon) \equiv \frac{1}{V} \sum_{\mathbf{k}} \delta\left(\varepsilon-\varepsilon_{\mathbf{k}}\right)$ is the density of states of the band, $d$ is the dimension of the system, $V$ is its volume, and $C=2 \pi\left|\Omega_{e}\right|^{2} / \hbar$. As we use the local density approximation, the Raman rate per particle only depends on the particle number and the trapping potential through the characteristic particle number $\rho=N\left(V_{T} / D\right)^{d / 2}$, where $N$ is the total number of atoms in the hyperfine mixture $\left(|1\rangle,\left|1^{\prime}\right\rangle\right)$. We are left with only three fitting parameters: the temperature $T$; the chemical potential at the center of the trap $\mu_{o}$; and an overall multiplicative factor. The second fitting function is the simplified expression

$$
R_{\pi / a}(\tilde{\omega}) \propto g_{v}(\hbar \tilde{\omega} / 2) e^{\left(\hbar \tilde{\omega} / 2 k_{B} T\right)} .
$$

This approximate expression, which has only temperature and a prefactor as fitting parameters, is only valid in certain parameter regimes; however, it has the advantage of being very simple.

In the following, we show that the values of temperature and central chemical potential obtained through this fitting procedure agree very well with the initial system parameters. This means that each Raman spectrum is to a good extent uniquely defined by its temperature and chemical potential in both two and three dimensions.

\section{Noninteracting fermions in two dimensions}

Let us first look at Raman spectra for a two-dimensional system with fixed characteristic particle number $\rho=2$ and varying temperatures. These spectra are shown in Fig. 4. From this figure, one can see that all spectra can be broken down into two parts. For large $\tilde{\omega}$, each spectrum is characterized by a signal of large amplitude whose shape depends on the system parameters while, for small $\tilde{\omega}$, each spectrum presents

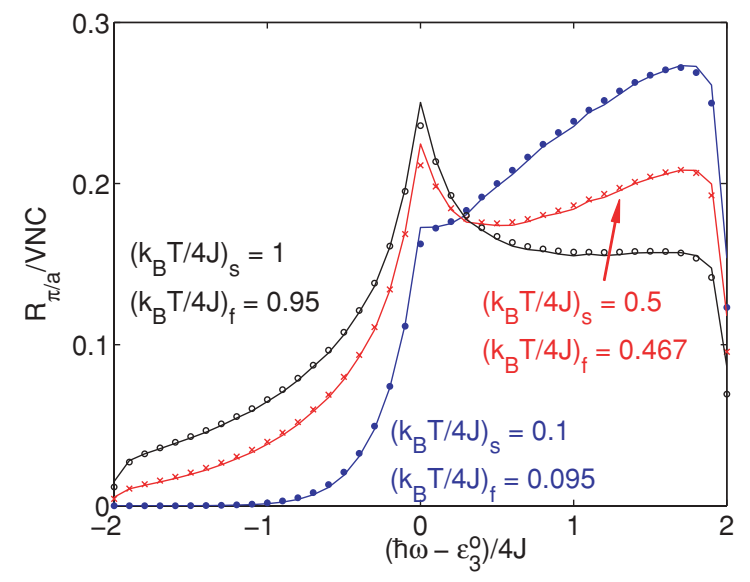

FIG. 4. (Color online) Raman spectra for three different temperatures $\left(k_{B} T / 4 J=0.1,0.5\right.$, and 1$)$ at the characteristic particle number $\rho=2$ in a two-dimensional system; $\left(k_{B} T / 4 J\right)_{s}$ is the exact temperature while $\left(k_{B} T / 4 J\right)_{f}$ is obtained by fitting the spectra to Eq. (8).

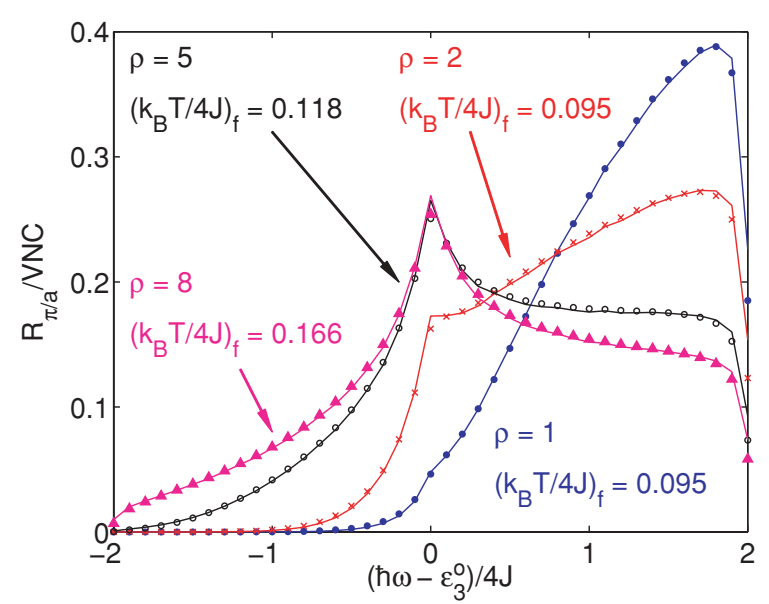

FIG. 5. (Color online) Raman spectra for four different characteristic particle numbers $\rho=1,2,5,8$ at fixed temperature $k_{B} T / 4 J=0.1$ in a two-dimensional system; $\left(k_{B} T / 4 J\right)_{f}$ is obtained by fitting the spectra to Eq. (8). Note that the case where the fit does not provide an accurate determination $(\rho=8)$ corresponds to an almost filled band at the trap center, with few available thermally excited states.

a tail whose shape is mainly set by temperature. The peak or discontinuity at $\tilde{\omega}=0$ is due to the Van Hove singularity of the square lattice density of states. The presence of sharp edges at $\hbar \tilde{\omega} / 4 J=\{-2,2\}$ is due to the abrupt ends of the square lattice density of states. At low temperatures, most of the spectral weight is located at $\tilde{\omega}>0$ with a sharp step around $\tilde{\omega} \approx 0\left(\mathrm{cf} . k_{B} T / 4 J=0.1\right)$. As the system temperature is increased, some weight is transferred into the tail and the step broadens considerably. This broadening stems from the smoothening of the Fermi function with increasing temperature. For high temperatures, the left end of the spectrum becomes sharp as it is cut by the edge of the density of states.

Figure 5 shows the dependence of Raman spectra with varying characteristic particle numbers at a fixed temperature. For small $\rho$, most of the weight is located in the bulk of the spectrum $(\tilde{\omega}>0)$, whereas, at higher $\rho$, more and more weight shifts into the tail. For very large characteristic particle number, the left edge of the spectrum is located at the end of the $\tilde{\omega}$ window allowed by the support of the density of states and not where the Fermi function goes to zero.

From the preceding descriptions, it is clear that the Raman spectra strongly depend on temperature and particle density. We exploit this strong dependence by fitting these Raman spectra to an integrated version of Eq. (6):

$$
\frac{R_{\pi / a}^{2 D}(\tilde{\omega})}{N} \propto \frac{T}{\rho} g_{v}(\hbar \tilde{\omega} / 2) \ln \left[1+e^{\left(\hbar \tilde{\omega} / 2+\mu_{o}\right) / k_{B} T}\right] .
$$

From this fit, we extract the temperature and central chemical potential of a two-dimensional system. The values obtained agree very well with the initial system parameters. We summarize the accuracy of the fitted parameters in Fig. 6. In the upper panel, we see that, aside from small deviations, the temperature can be determined very accurately by this procedure and is in most cases well within $10 \%$ of its true value. The middle panel shows that the chemical potential in the center of the trap can also be evaluated. Even though 

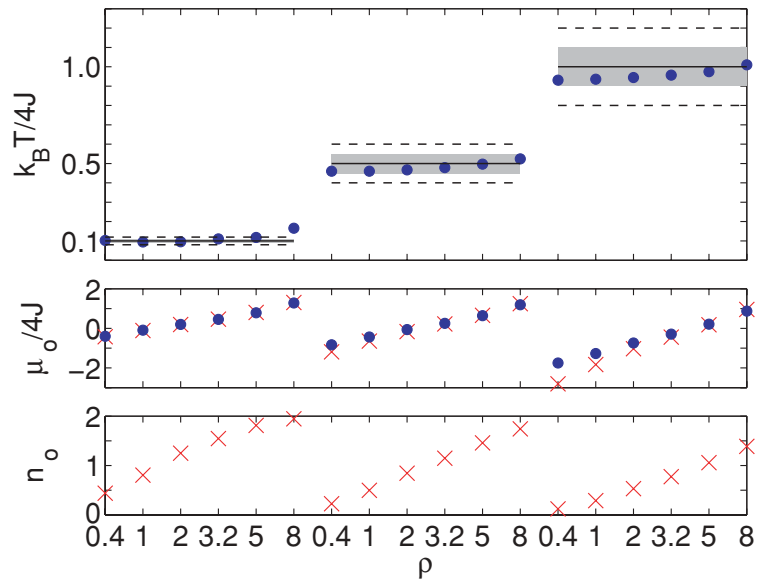

FIG. 6. (Color online) Accuracy of detected temperatures and central chemical potentials. These detected values were obtained by fitting each full spectrum with Eq. (8). Upper panel: detected temperatures are denoted by (blue) dots and exact temperatures by solid (black) lines. Each shaded region corresponds to a $10 \%$ range centered on the exact temperature and contains most fitted points. The regions delimited by dashed lines correspond to a $20 \%$ range. Middle panel: central chemical potentials are denoted by (blue) dots, exact values by (red) " $\times$ ". Lower panel: density at the center of the trap, $n_{o}$.

its accuracy is not as good as for temperature, it still agrees to within $20 \%$ for most of the simulated systems. Therefore, from the knowledge of experimentally measurable Raman spectra, we can accurately determine the temperature and obtain a good estimate of the chemical potential at the center of the trap for a free fermionic gas confined to a two-dimensional optical lattice. Let us point out that here we assumed that the hopping amplitude in the optical lattice, $J$, is known. However, as explained earlier, $J$ sets the support of the spectra at high fillings and can therefore be experimentally detected as a bonus by Raman spectroscopy measurements.

In many cases a much simpler fitting procedure can already give very good results for the temperature. This simplified method focuses on the behavior of the low-frequency part of the Raman spectrum tail. In Fig. 7, the Raman spectrum is shown on a logarithmic scale in order to emphasize its tails. Looking back at the analytical expression given by Eq. (8), we see that if $e^{\left(\hbar \tilde{\omega} / 2+\mu_{o}\right) / k_{B} T}$ is small the Raman signal can be approximated by

$$
R_{\pi / a}^{2 D}(\tilde{\omega}) \propto g_{v}(\hbar \tilde{\omega} / 2) e^{\left(\hbar \tilde{\omega} / 2 k_{B} T\right)}
$$

This simplified expression can be used as long as its validity extends over a sufficiently large region of measurable signal, that is, if $e^{\left(\hbar \tilde{\omega} / 2+\mu_{o}\right) / k_{B} T} \ll 1$ for a wide range of $\hbar \tilde{\omega} / 4 J \in$ $[-2 ; 2]$. This condition is most easily fulfilled for small or even negative $\mu_{o}$. Hence, this simplified fitting procedure holds best for small and intermediate characteristic densities. These limitations are quite apparent in Fig. 7. For example, increasing the characteristic density increases the signal in the tail but drastically reduces the region over which the fitting procedure works. For $\rho=5$, even at $k_{B} T / 4 J=0.1$, only a very small region is left. The same behavior is also observed at larger temperatures. In fact, the good fitting region completely drops off the spectrum at large temperatures and large characteristic



FIG. 7. (Color online) Raman spectra in logarithmic scale for four different characteristic particle numbers $(\rho=1,2,5,8)$ at fixed temperature $k_{B} T / 4 J=0.1$ in a two-dimensional system; $\left(k_{B} T / 4 J\right)_{f}$ is obtained by fitting the tails of the spectra to Eq. (9).

densities as this region would appear outside $\hbar \tilde{\omega} / 4 J \in[-2 ; 2]$, the range permitted by the density of states. The resulting accuracy of the simplified fitting procedure is summarized for different system parameters in Fig. 8. Here the simplified fit works very well for the lowest values of $\rho$, whereas for larger characteristic densities the temperature is overestimated.

\section{Noninteracting fermions in three dimensions}

Let us now look at Raman spectra for atoms confined to a cubic lattice. For fixed characteristic particle number and various temperatures, typical signals are shown in Fig. 9. At low temperatures, these spectra show a main peak and a tail that quickly goes to zero. For increasing temperatures, the structure of the cubic density of states becomes more apparent as weight shifts toward the tail. This change in the shape of the Raman spectrum is due to the Fermi function, whose spread increases with temperature. In Fig. 10 the evolution

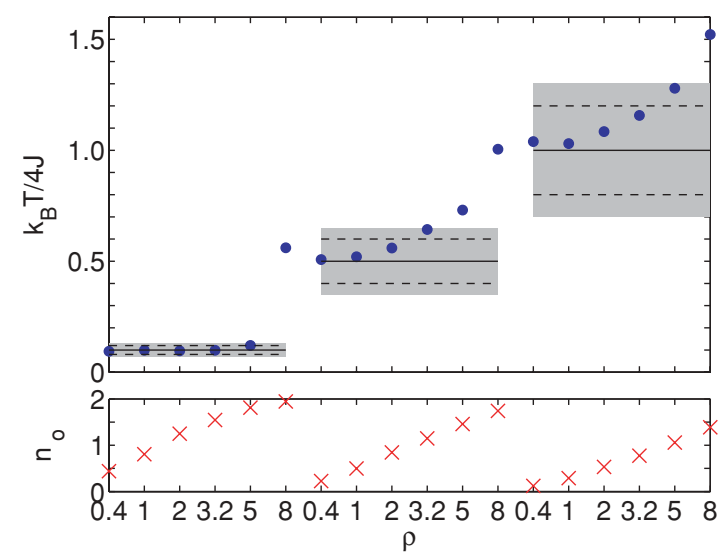

FIG. 8. (Color online) Accuracy of detected temperatures obtained by fitting the tail of each spectrum with Eq. (9). Upper panel: detected temperatures are denoted by (blue) dots and exact temperatures by solid (black) lines. Each shaded region corresponds to a $30 \%$ range centered on the exact temperature and contains most fitted points. The regions delimited by dashed lines correspond to a $20 \%$ range. Lower panel: density at the center of the trap. 




FIG. 9. (Color online) Raman spectra for three different temperatures $\left(k_{B} T / 6 J=0.1,0.5,1\right)$ at a fixed characteristic particle number $\rho=2.5$ in three dimensions; $\left(k_{B} T / 6 J\right)_{s}$ is the exact temperature while $\left(k_{B} T / 6 J\right)_{f}$ is obtained by fitting each spectrum up to its peak with Eq. (10).

of the spectra with increasing characteristic particle number, $\rho$, is shown at low temperature. For intermediate filling, the Raman spectrum presents its characteristic tail whose size depends strongly on temperature. At very large filling, the tail cannot be followed until its end as the Raman spectrum is limited by the frequency window allowed by the cubic density of states.

In three dimensions, we can also extract the temperature from the measured signal. In this case, the Raman rate in the continuum approximation is given by

$$
\frac{R_{\pi / a}^{3 \mathrm{D}}(\tilde{\omega})}{N} \propto \frac{g_{v}(\hbar \tilde{\omega} / 2)}{\rho} \int_{-\infty}^{\mu_{o}} d \mu \frac{\left(\mu_{o}-\mu\right)^{1 / 2}}{1+e^{-(\hbar \tilde{\omega} / 2+\mu) /\left(k_{B} T\right)}} .
$$

By fitting three-dimensional spectra with Eq. (10), we checked that the temperature and central chemical potential are, to a good degree, uniquely defined for a given spectrum. The quality of the extracted temperature and central chemical potential values are summarized in Fig. 11. We find very good agreement between the input and extracted temperatures if the

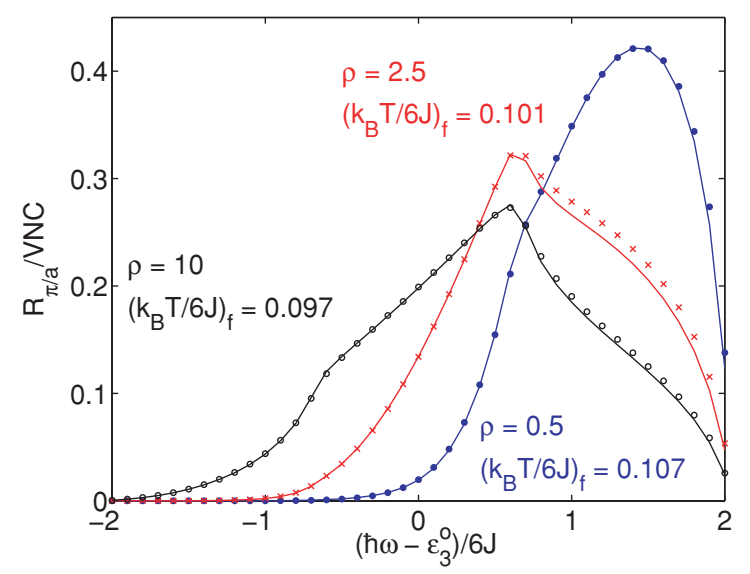

FIG. 10. (Color online) Raman spectra for three different characteristic particle numbers $(\rho=0.5,2.5,10)$ at fixed temperature $k_{B} T / 6 J=0.1$ in three dimensions; $\left(k_{B} T / 6 J\right)_{f}$ is obtained by fitting each spectrum up to its peak with Eq. (10).
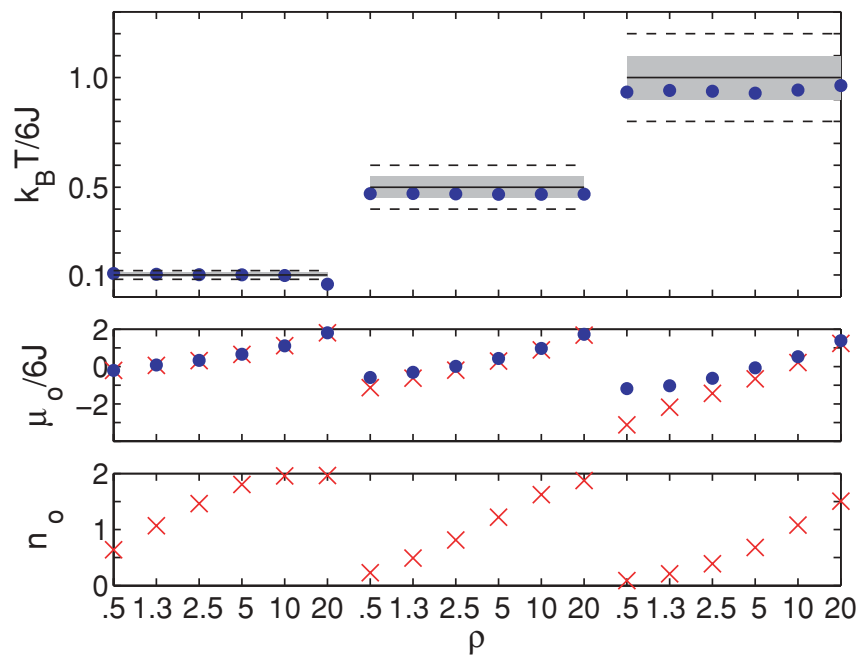

FIG. 11. (Color online) Accuracy of detected temperatures and central chemical potentials. These detected values were obtained by fitting each spectrum up to its peak with Eq. (10). Upper panel: detected temperatures are denoted by (blue) dots and exact temperatures by solid (black) lines. Each shaded region corresponds to a $10 \%$ range centered on the exact temperature and contains most fitted points. The regions delimited by dashed lines correspond to a $20 \%$ range. Middle panel: detected central chemical potentials are denoted by (blue) dots, exact values by (red) " $\times$ ". Lower panel: density at the center of the trap.

fit is done from $\tilde{\omega}_{\min }$ to the $\tilde{\omega}$ value corresponding to the peak of the spectrum. However, fitting over the whole spectrum is not as successful because more importance is given to the rightmost portion of the signal, which is not as sensitive to temperature as the tail. Using the reduced fitting range, the temperature can be determined to within $10 \%$ uncertainty. The chemical potential at the center of the trap can also be determined. However, the agreement between the input and extracted values decreases with increasing temperature.

Considering only the tail of the spectrum, a simplified fitting procedure can also be used to evaluate the temperature of a three-dimensional gas. Looking back at the analytical expression given by Eq. (10), we see that if $e^{\left(\tilde{\omega} / 2+\mu_{o}\right) / k_{B} T}$ is small [30] the Raman signal can be approximated by

$$
R_{\pi / a}^{3 D}(\tilde{\omega}) \propto g_{v}(\hbar \tilde{\omega} / 2) e^{\left(\hbar \tilde{\omega} / 2 k_{B} T\right)} .
$$

As in the two-dimensional case, we expect this expression to be accurate for small or even negative values of $\mu_{o}$ and small $\hbar \tilde{\omega} / 6 J \in[-2 ; 2]$. In Fig. 12, we apply this simplified fitting method to spectra with various characteristic particle numbers. For small values $(\rho=0.5,2.5)$, the fit works nicely over a wide range of frequencies. In contrast, for larger values of $\rho$, the range over which the simplified expression can be fitted becomes very small or nonexistent. In Fig. 13, the extracted temperatures are compared to the input temperatures. As expected, the procedure works well for small and intermediate characteristic particle numbers. In contrast, for large values of $\rho$, this method overestimates the system temperature.

Finally, to conclude this section, we need to point out that the frequency resolution attainable in experiments may not be as good as assumed here. Therefore, we checked that the fitting procedure still works for a reduced frequency resolution by 




FIG. 12. (Color online) Raman spectra in logarithmic scale for three different characteristic particle numbers $(\rho=0.5,2.5,10)$ at fixed temperature $k_{B} T / 6 J=0.1$ in three dimensions; $\left(k_{B} T / 6 J\right)_{f}$ is obtained by fitting the tails of the spectra to Eq. (11).

binning the simulated spectra. For two-dimensional systems, the temperature extraction method still works surprisingly well. The temperature can be determined very accurately even if only a few points are left on the spectrum $(\hbar \Delta \tilde{\omega} / 4 J=0.8)$. In three dimensions, the fitting procedure still works for a frequency resolution of about $\hbar \Delta \tilde{\omega} / 6 J=0.4$.

\section{B. Fermions with (moderate) interactions: Thermometry from the wings of the density profile}

When atoms in the $\left(|1\rangle,\left|1^{\prime}\right\rangle\right)$ mixture interact via a finite interaction strength $U$, the structure of the Raman spectrum changes significantly due to weight redistribution in the spectral function (cf. Sec. IV). However, up to intermediate interaction strengths, the low-density region on the periphery

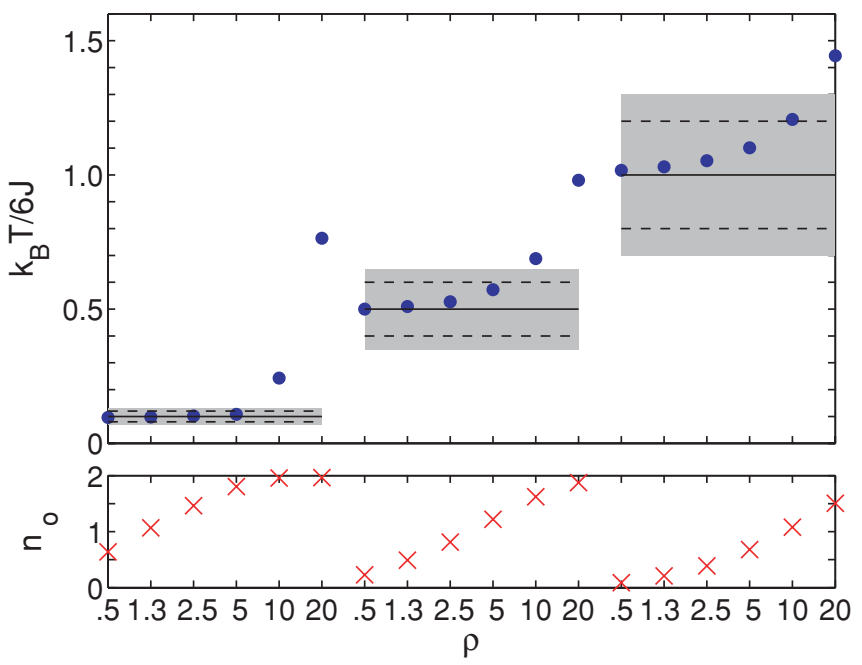

FIG. 13. (Color online) Accuracy of detected temperatures obtained by fitting the tail of each spectrum with Eq. (11). Upper panel: detected temperatures are denoted by (blue) dots and exact temperatures by solid (black) lines. Each shaded region corresponds to a $30 \%$ range centered on the exact temperature and contains most fitted points. The regions delimited by dashed lines correspond to a $20 \%$ range. Lower panel: density at the center of the trap.



FIG. 14. (Color online) Density cut through $(x, 0,0)$ for an interacting system at $U / 6 J=2, k_{B} T / 6 J=0.1$, and $\rho=8.9$. Below $n=0.3$, the interacting and Hartree-corrected density profiles agree quite well. The shaded region $(x>30)$ corresponds to the probed area; this region contains $0.4 \%$ of the total atom number.

of the trap is still well described by a system of noninteracting fermions. Thus, this region can be used to extract the gas temperature, assuming the system is in thermal equilibrium. The experimental feasibility of the detection of a small boundary region has been shown using radio-frequency spectroscopy for an imbalanced Fermi mixture and has been used to detect the temperature in the absence of an optical lattice potential [31]. In contrast to our proposal, the boundary region in that case was only occupied by the majority component so the Fermi gas in the wings was clearly noninteracting. Figures 14 and 15 show density profiles, obtained from dynamical mean-field calculations, for two three-dimensional interacting systems. We compare these profiles to those calculated by using local density approximation and the simple Hartree approximation for the relation between the density $n$ and the chemical potential $\mu$. The Hartree approximation simply amounts to inverting the relation: $\mu=\mu_{\mathrm{U}=0}(n)+\frac{U}{2} n$, where $\mu_{\mathrm{U}=0}(n)$ is the chemical potential of the free system for a given density $n$. As one can see from these figures, the interacting and

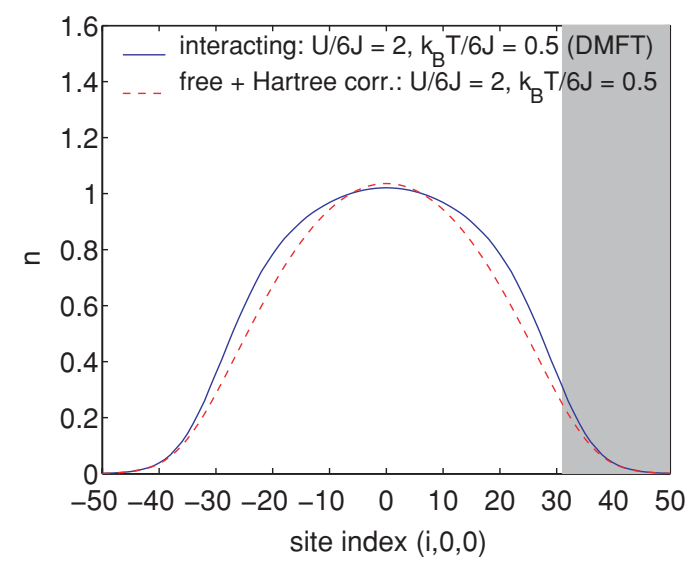

FIG. 15. (Color online) Density cut through $(x, 0,0)$ for an interacting system at $U / 6 J=2, k_{B} T / 6 J=0.5$, and $\rho=8.9$. Below $n=0.3$, the interacting and Hartree-corrected density profiles agree quite well. The shaded region $(x>31)$ corresponds to the probed area; this region contains $1.4 \%$ of the total atom number. 


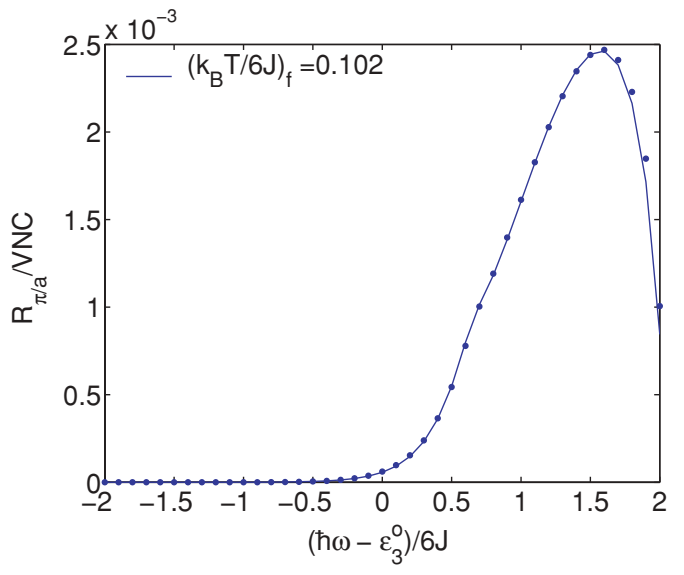

FIG. 16. (Color online) Raman spectrum obtained by only collecting signal from the low-density region shown in Fig. 14. The system exact temperature is $k_{B} T / 6 J=0.1$ while the detected temperature obtained through a fit of Eq. (12) is $\left(k_{B} T / 6 J\right)_{f}=0.102$.

Hartree-approximated profiles agree quite well for $n<0.3$, whereas the profiles are considerably different for larger densities. Hence, by only probing the region at the periphery of the trap, we can detect the system temperature as the atoms at these locations are still described by a quasi-non-interacting model. The validity of this approximation is further evidenced in Sec. IV. Two examples of Raman spectra obtained by collecting Raman signals coming from one of the six "semispherical" regions of low density are shown in Figs. 16 and 17. These Raman spectra are simulated using Eq. (5), where the sum over positions is limited to one of the six low-density regions and the central chemical potential, $\mu_{o}$, is the one setting the correct atom number in the interacting system. To show that the temperature can still be well detected in this limit, we fit these spectra using the continuum Raman expression:

$$
\begin{aligned}
\frac{R_{\pi / a}(\tilde{\omega})}{N} \propto & \frac{g_{v}(\hbar \tilde{\omega} / 2)}{\rho} \int_{0}^{\frac{\pi}{2}} \int_{\frac{\alpha}{\cos ^{2} \phi}}^{\infty} d x \sin \phi d \phi \\
& \times \frac{\sqrt{x}}{1+e^{-\left(\hbar \tilde{\omega} / 2+\mu_{o}-x\right) /\left(k_{B} T\right)}} .
\end{aligned}
$$

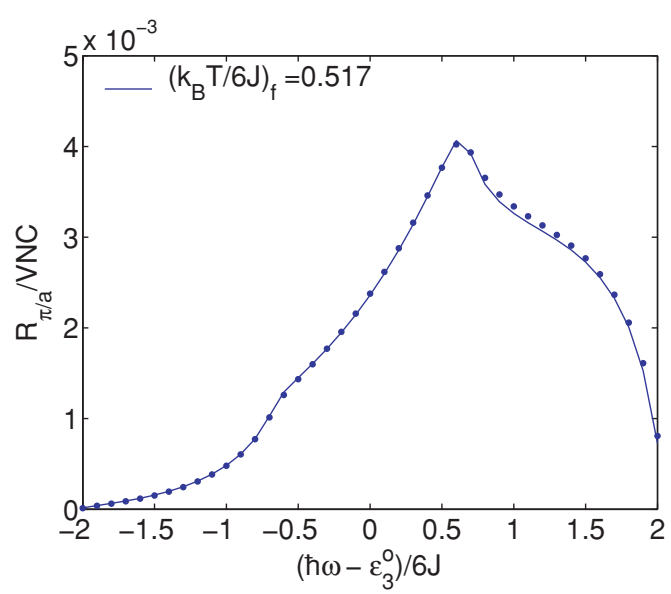

FIG. 17. (Color online) Raman spectrum obtained by only collecting signal from the low-density region shown in Fig. 15. The system exact temperature is $k_{B} T / 6 J=0.5$ while the detected temperature obtained through a fit of Eq. (12) is $\left(k_{B} T / 6 J\right)_{f}=0.517$.
In this expression, where the spatial integral is limited to the probed region, there are only three fitting parameters: the temperature, the central chemical potential, and $\alpha$, a parameter related to the size of the probed region [32]. As shown in Figs. 16 and 17, the system temperature can be measured successfully using this procedure.

\section{SPECTRA FOR INTERACTING FERMIONS: FROM STRONGLY CORRELATED FERMI LIQUIDS TO MOTT INSULATORS}

In this section we discuss the structure of the Raman spectrum in different strongly correlated states. We present general considerations based on the separate spectral contributions of quasiparticle excitations and of incoherent high-energy excitations. These considerations are illustrated by explicit calculations for the Hubbard model with repulsive interactions, treated in the framework of dynamical mean-field theory (DMFT) [33]. The DMFT calculations are performed using the numerical renormalization group method as an impurity solver [34]. The weakly correlated regime, strongly correlated Fermi liquid, and Mott-insulating regimes are discussed.

For simplicity, we focus on a homogeneous system, corresponding to Raman spectroscopy being performed in a local manner [23] and probing deep inside the bulk of a certain quantum state to avoid the influence of a neighboring state with different character [35]. We also restrict our discussion to the paramagnetic phase, and the calculations are performed at zero temperature (although some qualitative remarks are made on finite-temperature effects). The possible use of Raman spectroscopy to detect and investigate the magnetically ordered phase is left for future work.

We focus in this section on zero momentum transfer, $\mathbf{q}=0$. This is in contrast to the previous section, in which we used $\mathbf{q}=\pi / a$ in order to spread the signal as much as possible to probe thermally excited states. Here, on the contrary, we want to separate and resolve the different spectral features (e.g., quasiparticles and Hubbard bands) as well as possible, and for this $\mathbf{q}=0$ is more favorable [36]. We focus both on the momentum-resolved spectrum (i.e., after time of flight) and on the momentum-integrated signal (the latter being easier to achieve experimentally in the lattice) related to the spectral function $A(\mathbf{k}, \hbar \nu)$ [37] by

$$
\begin{aligned}
R_{\mathbf{q}=0}(\mathbf{k}, \omega)= & C n_{F}\left(\varepsilon_{3}^{o}-\hbar \omega+\varepsilon_{\mathbf{k}}-\mu\right) \\
& \times A\left(\mathbf{k}, \varepsilon_{3}^{o}-\hbar \omega+\varepsilon_{\mathbf{k}}-\mu\right), \\
R_{\mathbf{q}=0}(\omega)= & C \int d \mathbf{k} n_{F}\left(\varepsilon_{3}^{o}-\hbar \omega+\varepsilon_{\mathbf{k}}-\mu\right) \\
& \times A\left(\mathbf{k}, \varepsilon_{3}^{o}-\hbar \omega+\varepsilon_{\mathbf{k}}-\mu\right) .
\end{aligned}
$$

We recall that, in these expressions, $\varepsilon_{3}^{o}+\varepsilon_{\mathbf{k}}$ is the dispersion of the outcoupled state $|3\rangle$, while $\mu$ is the chemical potential of the interacting $\left(|1\rangle,\left|1^{\prime}\right\rangle\right)$ mixture. From Eq. (1), the prefactor reads $C=2 \pi\left|\Omega_{e}\right|^{2} / \hbar$ (we note that the Wannier matrix element $W_{\mathbf{k}}^{\mathbf{q}=0}=1$, assuming the same lattice potential for state $|1\rangle$ and $|3\rangle)$.

When specializing for DMFT calculations, the self-energy, $\Sigma$, only depends on frequency, so the spectral function $\pi A(\mathbf{k}, \hbar v) \equiv-\operatorname{Im}\left\{1 /\left[\hbar v+\mu-\varepsilon_{\mathbf{k}}-\Sigma\left(\hbar v+\mathrm{i} 0^{+}\right)\right]\right\}$depends 
on momentum through $\varepsilon_{\mathbf{k}}$ only. In this case, the momentum integration can be replaced by an integration over the density of states, $g_{v}(\varepsilon)$, associated with the dispersion $\varepsilon_{\mathbf{k}}$ (for simplicity, the following DMFT calculations are performed for a semicircular density of states):

$$
\begin{aligned}
R_{\mathbf{q}=0}(\omega)= & C V \int d \varepsilon g_{v}(\varepsilon) n_{F}\left(\varepsilon_{3}^{o}-\hbar \omega+\varepsilon-\mu\right) \\
& \times A\left(\mathbf{k}, \varepsilon_{3}^{o}-\hbar \omega+\varepsilon-\mu\right) .
\end{aligned}
$$

At $T=0$, the Fermi function in these expressions limits the integration domain to momenta such that $\varepsilon<\mu+\hbar \omega-\varepsilon_{3}^{o}$.

We note that these spectra obey the following sum rules, valid at arbitrary temperature $T$ :

$\int d \omega R_{\mathbf{q}=0}(\mathbf{k}, \omega)=C n_{1}(\mathbf{k}), \quad \int d \omega R_{\mathbf{q}=0}(\omega)=C N / 2$.

Hence, the total intensity of the momentum-resolved signal is proportional to the momentum distribution $n_{1}(\mathbf{k}) \equiv\left\langle c_{1 \mathbf{k}}^{\dagger} c_{1 \mathbf{k}}\right\rangle$ of particles of type $|1\rangle$ in the system, while the total intensity of the momentum-integrated signal is proportional to the total number of particles $N / 2$ in state $|1\rangle$. In these expressions, the frequency integration is over the whole range of frequencies where the signal is nonzero (this range is bounded from below, as shown later).

Let us first discuss the shape of the Raman spectrum in the simple case of a noninteracting system for which $A(\mathbf{k}, \hbar v)=$ $\delta\left(\hbar \nu+\mu-\varepsilon_{\mathbf{k}}\right)$. We obtain in this case

$$
\begin{gathered}
R_{\mathbf{q}=0}^{U=0}(\mathbf{k}, \omega)=C \delta\left(\hbar \omega-\varepsilon_{3}^{o}\right) n_{F}\left(\varepsilon_{\mathbf{k}}-\mu\right), \\
R_{\mathbf{q}=0}^{U=0}(\omega)=C \frac{N}{2} \delta\left(\hbar \omega-\varepsilon_{3}^{o}\right) .
\end{gathered}
$$

Hence, at $\mathbf{q}=0$ and in the absence of interactions, Raman transitions only exists at the frequency $\hbar \omega=\varepsilon_{3}^{o}$. This is due to the assumption that the dispersions for the atoms in the $\left(|1\rangle,\left|1^{\prime}\right\rangle\right)$ mixture and in the outcoupled state $|3\rangle$ are the same (cf. Fig. 3). At $T=0$, the momentum-resolved signal is nonzero only for momenta inside the Fermi surface $\varepsilon_{\mathbf{k}}<\mu$ because this spectroscopy probes only occupied states. At $T \neq 0$, the signal extends beyond the Fermi surface because of thermal broadening according to the Fermi function. These simple considerations are nicely illustrated by the spectra displayed in Figs. 18 and 19. These results correspond to the Hubbard model with a very low density of particles per site ( $n \approx 0.18$, dilute system) and a very high density of particles per site ( $n \approx 1.82$, or low density of holes in a band insulator), respectively. Despite the fact that these DMFT calculations were made for a rather high value of $U / D=3.5$ (with $D$ the half bandwidth), the system is in effect weakly correlated because the density of particles (or holes) is small. This is clearly seen from the displayed momentum-resolved spectral functions [Figs. 18(b) and 19(b)] as they are weakly modified as compared to the noninteracting case. Both spectra show a very sharp peak which disperses essentially according to the free dispersion $\varepsilon_{\mathbf{k}}$ (only a shift in position is shown). Hence, the Raman spectra are closely following the noninteracting behavior: the momentum-integrated spectra [Figs. 18(c) and 19(c)] are sharply peaked, and the momentum-resolved Raman spectra [Figs. 18(a) and 19(a)] have very little momentum dispersion (in contrast to the spectral function itself) and are
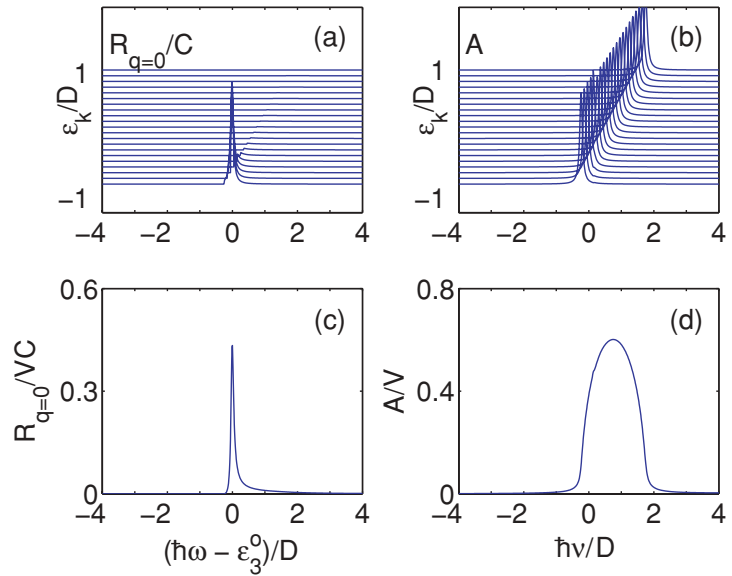

FIG. 18. (Color online) Spectra for a low-density liquid $(\mu / D=$ $-0.75, U / D=3.5$, and $n \approx 0.18$ ): (a) momentum-resolved Raman spectrum (in arbitrary units), (b) momentum-resolved spectral function (in arbitrary units), (c) momentum-integrated Raman spectrum, and (d) momentum-integrated spectral function.

suppressed for momenta outside the Fermi surface. Due to this narrow momentum dispersion, a very sharp peak occurs in the momentum-integrated Raman spectra [Figs. 18(c) and 19(c)] (the peak position is discussed later). Let us emphasize that these findings further support the detection scheme for interacting particles presented in Sec. III B, which relies on the assumption that in the low-density regions the spectral function behaves like the one of the noninteracting particles.

We now turn to spectra in which effects of strong correlations become more pronounced. In order to discuss these spectra on a general basis, we can separate the spectral function into a contribution from quasiparticles and a contribution from high-energy incoherent excitations:

$$
A(\mathbf{k}, \hbar v)=A_{\mathrm{qp}}(\mathbf{k}, \hbar v)+A_{\text {inc }}(\mathbf{k}, \hbar v)
$$
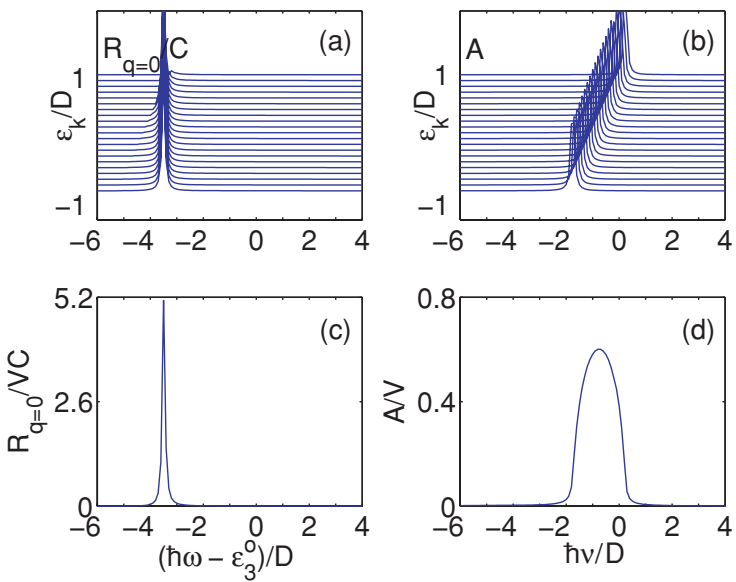

FIG. 19. (Color online) Spectra for a high-density liquid $(\mu / D=$ 4.25, $U / D=3.5, n \approx 1.82$ ): (a) momentum-resolved Raman spectrum (in arbitrary units), (b) momentum-resolved spectral function (in arbitrary units), (c) momentum-integrated Raman spectrum, and (d) momentum-integrated spectral function. 
The quasiparticle contribution can be appropriately described, at low excitation energies and close to the Fermi surface, by a sharply peaked Lorentzian:

$$
A_{\mathrm{qp}}(\mathbf{k}, \hbar v) \simeq \frac{Z_{\mathbf{k}}}{\pi} \frac{\Gamma_{\mathbf{k}}}{\left[\hbar v-\left(\varepsilon_{\mathbf{k}}^{\mathrm{qp}}-\mu\right)\right]^{2}+\Gamma_{\mathbf{k}}^{2}} .
$$

In this expression, $\varepsilon_{\mathbf{k}}^{\mathrm{qp}}$ is the dispersion relation of quasiparticles, $\Gamma_{\mathbf{k}}$ is their inverse lifetime, and $Z_{\mathbf{k}}$ is the spectral weight associated with the contribution of quasiparticles to the total spectrum of single-particle excitations [38]. In a Fermi liquid, the quasiparticle excitations become long-lived coherent excitations as the Fermi surface is approached, corresponding to a sharp peak with width $\Gamma_{\mathbf{k}} \propto\left(\varepsilon_{\mathbf{k}}^{\mathrm{qP}}-\mu\right)^{2} \sim$ $\left(\mathbf{k}-\mathbf{k}_{F}\right)^{2}$.

To illustrate how quasiparticles contribute to the Raman spectrum, Fig. 20 displays the results of a DMFT calculation for the half-filled Hubbard model at $U / D=1.5$, which corresponds to a Fermi liquid in the intermediate correlation regime. The momentum-resolved spectral function [Fig. 20(b)] clearly displays a quasiparticle peak. This quasiparticle peak becomes sharp as the Fermi surface is reached (corresponding here to $\varepsilon_{\mathbf{k}_{\mathrm{F}}}=0$ ), while for momenta far from the Fermi surface only a broader incoherent contribution is seen. The momentum-resolved Raman spectrum [Fig. 20(a)] shows the same features below the Fermi level. However, the dispersion of the quasiparticle peak close to $\mathbf{k}_{\mathrm{F}}$ and the incoherent contribution far from the Fermi surface behave differently than in the spectral function. The momentumintegrated Raman spectrum [Fig. 20(c)] has a well-marked peak corresponding to quasiparticle contribution to the density of states, and a broader hump corresponding to incoherent excitations. In order to better understand these spectral features, we note that the contribution of quasiparticles
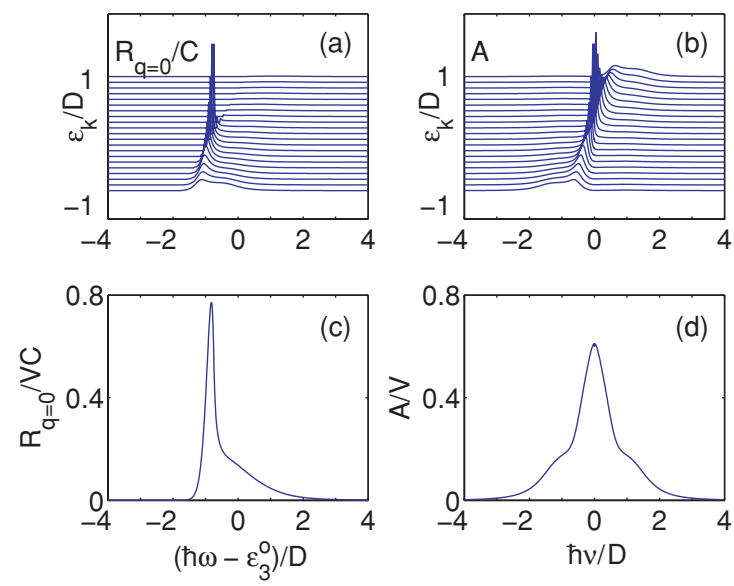

FIG. 20. (Color online) Spectra for a Fermi liquid with moderate correlations $(\mu / D=0.75, U / D=1.5, n=1)$ : (a) momentum-resolved Raman spectrum (in arbitrary units), (b) momentum-resolved spectral function (in arbitrary units), (c) momentum-integrated Raman spectrum, and (d) momentumintegrated spectral function. to the momentum-resolved Raman spectrum reads, using Eq. (19) in Eq. (13),

$$
\begin{aligned}
R_{\mathbf{q}=0}^{\mathrm{qp}}(\mathbf{k}, \omega) \simeq & C n_{F}\left(\varepsilon_{\mathbf{k}}^{\mathrm{qp}}-\mu\right) \frac{Z_{\mathbf{k}}}{\pi} \\
& \times \frac{\Gamma_{\mathbf{k}}}{\left[\hbar \omega-\varepsilon_{3}^{o}-\left(\varepsilon_{\mathbf{k}}-\varepsilon_{\mathbf{k}}^{\mathrm{qp}}\right)\right]^{2}+\Gamma_{\mathbf{k}}^{2}} .
\end{aligned}
$$

From this expression, it is clear that the quasiparticle peak in the momentum-resolved Raman spectrum disperses according to $\left(\hbar \omega-\varepsilon_{3}^{o}\right)_{\mathbf{k}}^{\mathrm{qp}}=\varepsilon_{\mathbf{k}}-\varepsilon_{\mathbf{k}}^{\mathrm{qp}} \sim\left(\mathbf{v}_{F}-\mathbf{v}_{F}^{\mathrm{qp}}\right) \cdot\left(\mathbf{k}-\mathbf{k}_{F}\right)+\cdots$. The last expression is valid for momenta near the Fermi surface and involves the difference between the actual Fermi velocity, $\mathbf{v}_{F}^{\mathrm{qp}}$, in the presence of interactions (related to the effective mass) and the bare Fermi velocity, $\mathbf{v}_{F}$. Indeed, the peak in the Raman signal is less dispersive [Fig. 20] than the one in the spectral function [dispersing as $\mathbf{v}_{F}^{\mathrm{qp}} \cdot\left(\mathbf{k}-\mathbf{k}_{F}\right)$ ]. In practice, since the dispersion $\varepsilon_{\mathbf{k}}$ of the outcoupled band is known, it is possible to extract $\varepsilon_{\mathbf{k}}^{\mathrm{qp}}$ directly from the Raman signal by plotting it as a function of $\hbar \omega-\varepsilon_{3}^{o}-\varepsilon_{\mathbf{k}}$ (as done in [6] in the continuum). As the quasiparticle peak becomes very sharp near the Fermi surface $\mathbf{k} \simeq \mathbf{k}_{F}$, those momenta dominate the momentum integration [at least for lattices with a nonsingular $\left.g_{v}(\varepsilon)\right]$. Hence, the momentum-integrated spectrum [Fig. 20(c)] has a quasiparticle peak located at $\left(\hbar \omega-\varepsilon_{3}^{o}\right)^{\mathrm{qP}}=\left\langle\varepsilon_{\mathbf{k}_{F}}\right\rangle-\mu \simeq$ $\mu_{U=0}-\mu$. In the first expression, $\left\langle\varepsilon_{\mathbf{k}_{F}}\right\rangle$ corresponds to a Fermi surface average. The second expression is valid when the Fermi surface is only mildly deformed by interactions, so that the Luttinger theorem (conservation of Fermi surface volume) implies that $\varepsilon_{\mathbf{k}_{F}}=\mu_{U=0}$, where $\mu_{U=0}$ is the chemical potential of the noninteracting system at the same density. This analysis accounts well for the location of the peak (at $\sim-\mu$ ) in the spectrum of Fig. 20(c) (which corresponds to half filling, so that $\mu_{U=0}=0$, while $\mu=U / 2=0.75 D$ ). The onset of Raman absorption in the momentum-integrated spectrum at $T=0$ corresponds to the restriction due to the Fermi function $\hbar \omega-\varepsilon_{3}^{o}>\varepsilon_{\mathbf{k}}-\mu$ and, hence, corresponds to a threshold frequency, $\left(\hbar \omega-\varepsilon_{3}^{o}\right)^{\text {th }}=-D-\mu$, again well obeyed in Fig. 20(c). We note that this absorption threshold corresponds to the transfer of states from the bottom of the band with $\mathbf{k}=0$ (i.e., occupied states well below the Fermi surface), as is known from radio-frequency spectroscopy $[4,5]$.

Having discussed a Fermi liquid in the regime of intermediate correlations, we turn to the opposite limit of a very strongly correlated system: a Mott insulator, as realized, for example, in the Hubbard model at half filling and for large interaction strength $(U / D=3.5$ in Fig. 21). There, quasiparticles are absent and the high-energy incoherent excitations correspond to the Hubbard "bands." The lower (upper) Hubbard band (LHB, resp. UHB) corresponds to the process of removing (adding) an atom on a singly occupied site. This corresponds to two peaks in the spectral function [Figs. 21(b) and 21(d)] at $\hbar \nu_{\mathbf{k}}^{\mathrm{LHB}}<0$ and $\hbar v_{\mathbf{k}}^{\mathrm{UHB}}>0$, separated by the Mott gap $\Delta_{g}$. Since the excitation energy from the ground state for removing a particle is $\mu$, the lower Hubbard band $\hbar \nu_{\mathbf{k}}^{\mathrm{LHB}}$ is centered at $\sim-\mu$. This band disperses over a bandwidth of order $D$ with a width $\Gamma_{\mathbf{k}}^{\mathrm{LHB}}$ of order $D$ itself. Hence, the excitation is "incoherent" in nature (except at momenta near the top of the band where the width is smaller, of order $D^{2} / U$ ). Similar 

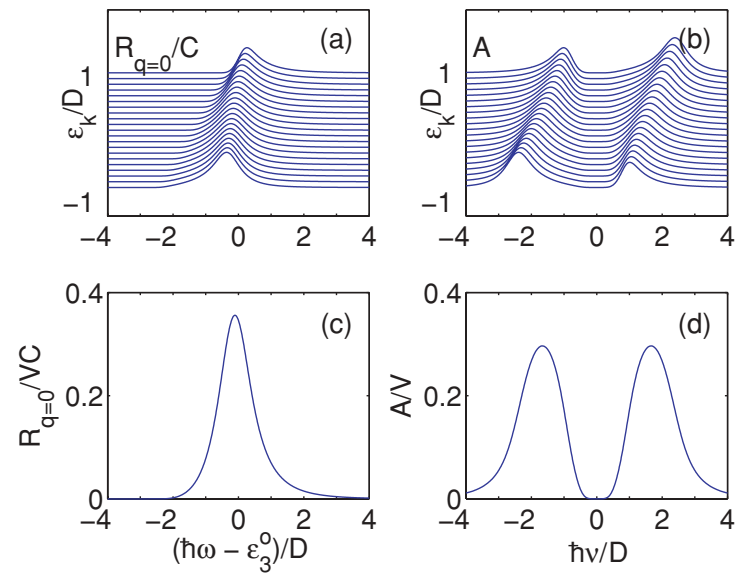

FIG. 21. (Color online) Spectra for a Mott insulator $(\mu / D=$ 1.75, $U / D=3.5, n=1$ ): (a) momentum-resolved Raman spectrum (in arbitrary units), (b) momentum-resolved spectral function (in arbitrary units), (c) momentum-integrated Raman spectrum, and (d) momentum-integrated spectral function.

considerations apply to the upper Hubbard band (centered at $\sim U-\mu$ ). The total (momentum-integrated) weight of the lower Hubbard band in the spectral function is proportional to $n / 2$, while that of the upper Hubbard band is proportional to $1-n / 2$.

At $T=0$, the lower Hubbard band is fully visible in the Raman spectrum, as seen in Figs. 21(a) and 21(c) (where $-\mu=-U / 2=-1.75 D)$. This lower band in the momentumresolved Raman spectrum is located at $\left(\hbar \omega-\varepsilon_{3}^{o}\right)_{\mathbf{k}}^{\mathrm{LHB}}=\varepsilon_{\mathbf{k}}-$ $\mu-\hbar v_{\mathbf{k}}^{\mathrm{LHB}}$. Since $\hbar v_{\mathbf{k}}^{\mathrm{LHB}}<0$, the lower Hubbard band is apparent for all momenta (in contrast to a quasiparticle peak, which is suppressed as the Fermi surface is crossed). As the momentum integration is dominated by $\varepsilon_{\mathbf{k}}=0$, and $\hbar \nu_{\mathbf{k}}^{\mathrm{LHB}}$ is centered at $-\mu$, the lower Hubbard band results in a peak in the momentum-integrated spectrum located at $(\hbar \omega-$ $\left.\varepsilon_{3}^{o}\right)^{\mathrm{LHB}} \simeq-\mu-(-\mu)=0$, which is clear from Fig. 21(c). The threshold for Raman absorption corresponds to $\left(\hbar \omega-\varepsilon_{3}^{o}\right)^{\text {th }}=$ $\min _{\mathbf{k}}\left[\varepsilon_{\mathbf{k}}-\hbar v_{\mathbf{k}}^{\mathrm{LHB}}\right]-\mu$. The minimum is usually realized for $\varepsilon_{\mathbf{k}}=-D$, so that $\left(\hbar \omega-\varepsilon_{3}^{o}\right)^{\text {th }}=-D-\hbar v_{\text {top }}^{\text {LHB }}-\mu$. At half filling, this reads $\left(\hbar \omega-\varepsilon_{3}^{o}\right)^{\text {th }}=-D+\Delta_{g} / 2-U / 2$, where $\Delta_{g}$ is the Mott gap. For temperatures comparable to or higher than the Mott gap, the upper Hubbard band becomes visible in Raman spectra at a location $\left(\hbar \omega-\varepsilon_{3}^{o}\right)^{\mathrm{UHB}} \simeq-U$. Concretely, imaging the integrated or full Hubbard bands would be very useful as it would not only give information on these incoherent excitations themselves, but also provide a method to extract the interacting strength $U$ and the gap size $\Delta_{g}$.

Finally, we display results for a strongly correlated Fermi liquid with a spectral function that simultaneously displays a central peak of quasiparticle excitations, as well as lower and upper Hubbard bands (Fig. 22, corresponding to a rather large coupling $U / D=3.5$ with $n=0.85$; i.e., to a strongly correlated Fermi liquid). The Raman spectra reveal both types of excitations, which also lead to two distinct features in the momentum-integrated Raman spectrum [Fig. 22(c)] at frequencies expected from the analysis above. We note that these two features generally have very different temperature dependences. As the temperature is raised, the quasiparticle
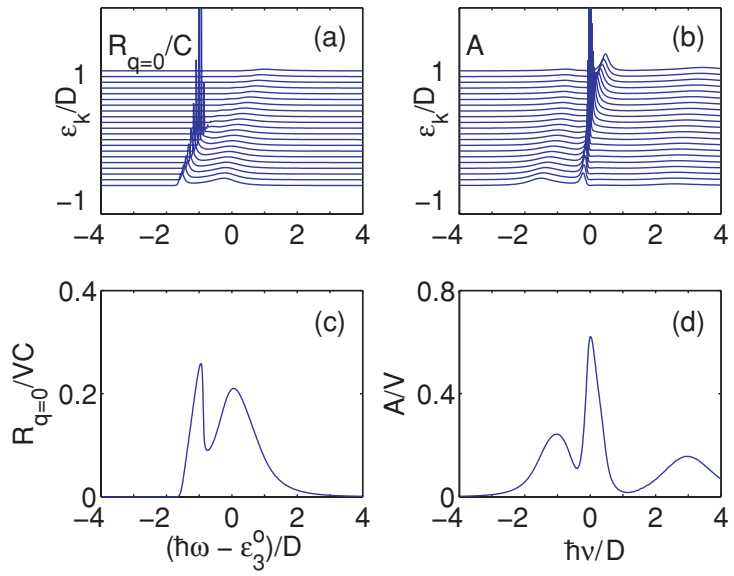

FIG. 22. (Color online) Spectra for a strongly correlated Fermi liquid $(\mu / D=0.75, \quad U / D=3.5, \quad n \approx 0.85)$ : (a) momentumresolved Raman spectrum (in arbitrary units), (b) momentumresolved spectral function (in arbitrary units), (c) momentumintegrated Raman spectrum, and (d) momentum-integrated spectral function.

peak is suppressed when temperature exceeds the quasiparticle coherence temperature, of order $Z_{\mathbf{k}_{F}} D$. In contrast, the lower Hubbard band starts losing weight (and the upper Hubbard band starts appearing) only at a higher temperature scale comparable to the gap scale.

In summary, Raman spectroscopy is a useful probe to explore various possible regimes of correlations. Broad Hubbard bands are seen in the incompressible Mott regime, while the additional observation of a quasiparticle peak at low temperature signals the formation of a strongly correlated Fermi liquid.

\section{CONCLUSION}

In this work, it was demonstrated that Raman spectroscopy is a versatile probe that can be used to measure the temperature of Fermi gases confined to optical lattices and to identify various signatures of strongly correlated fermionic phases. The proposed detection scheme, implementable with present technology, relies on transferring a portion of the atoms stored in the optical lattice potential to a third hyperfine state. This Raman rate can in principle be resolved in both frequency and momentum. We showed that momentum resolution is not required to accurately measure the temperature of free and weakly interacting fermionic atoms loaded into an optical lattice and that the detection can be done either locally or globally. We also demonstrated that detecting several features of strongly correlated liquids and Mott insulators such as quasiparticle peaks and Hubbard bands can be done using the same scheme without knowledge of the atom momentum. However, in the future, if momentum resolution is experimentally achieved in a lattice, the momentum-resolved Raman rate could provide valuable information on the level of correlation of fermionic cold-atom systems. Finally, we would like to point out that Raman spectroscopy can even be used to cool down fermionic atoms confined to an optical lattice as explained in [39]. 


\section{ACKNOWLEDGMENTS}

We are grateful to I. Bloch, I. Carusotto, J. Dalibard, M. Köhl, L. Perfetti, C. Salomon, and the Quantum Optics group of ETHZürich for stimulating discussions. We acknowledge support from the Triangle de la Physique, the Agence
Nationale de la Recherche (under contracts FABIOLA and FAMOUS), the Defense Advanced Research Projects Agency Optical Lattice Emulator program, the Fonds Québécois de la Recherche sur la Nature et les Technologies, and ANPCyT Grant No. 482/06.
[1] M. Köhl, H. Moritz, T. Stöferle, K. Günter, and T. Esslinger, Phys. Rev. Lett. 94, 080403 (2005).

[2] R. Jördens, N. Strohmaier, K. Günter, H. Moritz, and T. Esslinger, Nature 455, 204 (2008).

[3] U. Schneider, L. Hackermüller, S. Will, T. Best, I. Bloch, T. A. Costi, R. W. Helmes, D. Rasch, and A. Rosch, Science 322, 1520 (2008).

[4] W. Ketterle and M. Zwierlein, in Ultra-cold Fermi Gases, in Proceedings of the CLXYV International School of Physics "Enrico Fermi" (IOS, Amsterdam, 2007), Vol. 164, pp. 95-287.

[5] I. Bloch, J. Dalibard, and W. Zwerger, Rev. Mod. Phys. 80, 885 (2008).

[6] J. Stewart, J. Gaebler, and D. Jin, Nature 454, 744 (2008).

[7] S. Trotzky, L. Pollet, F. Gerbier, U. Schnorrberger, I. Bloch, N. Prokof'ev, B. Svistunov, and M. Troyer, e-print arXiv:0905.4882.

[8] D. M. Weld, P. Medley, H. Miyake, D. Hucul, D. E. Pritchard, and W. Ketterle, Phys. Rev. Lett. 103, 245301 (2009).

[9] C. Hung, X. Zhang, N. Gemelke, and C. Chin, e-print arXiv:0910.1382.

[10] D. McKay and B. DeMarco, e-print arXiv:0911.4143.

[11] T. Stöferle, H. Moritz, K. Günter, M. Köhl, and T. Esslinger, Phys. Rev. Lett. 96, 030401 (2006).

[12] M. Köhl, Phys. Rev. A 73, 031601(R) (2006).

[13] H. G. Katzgraber, A. Esposito, and M. Troyer, Phys. Rev. A 74, 043602 (2006).

[14] L. De Leo, C. Kollath, A. Georges, M. Ferrero, and O. Parcollet, Phys. Rev. Lett. 101, 210403 (2008).

[15] T.-L. Ho and Q. Zhou, e-print arXiv:0908.3015.

[16] J. Ruostekoski, C. J. Foot, and A. B. Deb, Phys. Rev. Lett. 103, 170404 (2009).

[17] X. Du, S. Wan, E. Yesilada, C. Ryu, and D. J. Heinzen, e-print arXiv:0704.2623.

[18] D. Clément, N. Fabbri, L. Fallani, C. Fort, and M. Inguscio, Phys. Rev. Lett. 102, 155301 (2009).

[19] P. T. Ernst, S. Götze, J. Krauser, K. Pyka, D.-S. Lühmann, D. Pfannkuche, and K. Sengstock, Nature Phys. 6, 56 (2010).
[20] S. Konabe, T. Nikuni, and M. Nakamura, Phys. Rev. A 73, 033621 (2006).

[21] P. B. Blakie, New J. Phys. 8, 157 (2006).

[22] T.-L. Dao, A. Georges, J. Dalibard, C. Salomon, and I. Carusotto, Phys. Rev. Lett. 98, 240402 (2007).

[23] T.-L. Dao, I. Carusotto, and A. Georges, Phys. Rev. A 80, 023627 (2009).

[24] T.-L. Dao, Ph.D. Thesis, École Polytechnique, 2008, [http:// imprimerie.polytechnique.fr/Theses/Files/DaoTungLam.pdf].

[25] Radio-frequency spectroscopy is also possible for the cases of vanishing momentum transfer.

[26] D. Jaksch, C. Bruder, J. I. Cirac, C. W. Gardiner, and P. Zoller, Phys. Rev. Lett. 81, 3108 (1998).

[27] W. Hofstetter, J. I. Cirac, P. Zoller, E. Demler, and M. D. Lukin, Phys. Rev. Lett. 89, 220407 (2002).

[28] In the case of a single frequency, the linear response is valid for a more restrictive time interval.

[29] For $V_{\text {lattice }} / E_{\mathbf{R}}=7$, only a small change of the overall amplitude is visible.

[30] If this condition is fulfilled for $\mu_{0}$, it is also fulfilled for the whole integration range.

[31] Y. Shin, C. Schunck, A. Schirotzek, and W. Ketterle, Nature 451, 689 (2008).

[32] $\alpha=V_{T} r_{\min }^{2}$, where $r_{\min }$ is the radial position at which $n=0.3$.

[33] A. Georges, G. Kotliar, W. Krauth, and M. J. Rozenberg, Rev. Mod. Phys. 68, 13 (1996).

[34] R. Bulla, T. A. Costi, and T. Pruschke, Rev. Mod. Phys. 80, 395 (2008).

[35] R. W. Helmes, T. A. Costi, and A. Rosch, Phys. Rev. Lett. 100, 056403 (2008).

[36] $\mathbf{q}=0$ can also be realized using radio-frequency spectroscopy.

[37] M. Imada, A. Fujimori, and Y. Tokura, Rev. Mod. Phys. 70, 1039 (1998).

[38] These quantities are related to the self-energy by the standard expressions $Z_{\mathbf{k}}=1 /\left(1-\partial \Sigma / \partial(\hbar v)_{v=0, \mathbf{k}}\right), \varepsilon_{\mathbf{k}}^{\mathrm{qp}}=Z_{\mathbf{k}}\left[\varepsilon_{\mathbf{k}}+(\mathbf{k}-\right.$ $\left.\left.\mathbf{k}_{F}\right) \cdot \nabla_{\mathbf{k}} \Sigma\right], \Gamma_{\mathbf{k}}=Z_{\mathbf{k}} \operatorname{Im}\left[\Sigma\left(\mathbf{k}, \varepsilon_{\mathbf{k}}^{\mathrm{qp}}-\mu\right)\right]$.

[39] A. Griessner, A. J. Daley, S. R. Clark, D. Jaksch, and P. Zoller, Phys. Rev. Lett. 97, 220403 (2006). 